\title{
Some aspects of global Lambda polarization in heavy-ion collisions
}

\author{
Xiao-Liang Xia ${ }^{a}$, Hui Li ${ }^{b}$, Qun Wang* \\ Department of Modern Physics, University of Science and Technology of China, Hefei, Anhui \\ 230026, China \\ ${ }^{a}$ E-mail: xiaxl@mail.ustc.edu.cn \\ ${ }^{b}$ E-mail: lihuil2@mail.ustc.edu.cn \\ ${ }^{c}$ E-mail: qunwang@ustc.edu.cn
}

\begin{abstract}
Large orbital angular momentum can be generated in non-central heavy-ion collisions, and part of it is expected to be converted into final particle's polarization due to the spin-orbit coupling. Within the framework of A Multi-Phase Transport (AMPT) model, we studied the vorticityinduced polarization of $\Lambda$ hyperons at the midrapidity region $|\eta|<1$ in Au-Au collisions at energies $\sqrt{s_{N N}}=7.7 \sim 200 \mathrm{GeV}$. Our results show that the global polarization decreases with the collisional energies and is consistent with the recent STAR measurements. This behavior can be understood by less asymmetry of participant matter in the midrapidity region due to faster expansion of fireball at higher energies. As another evidence, we discuss how much the angular momentum is deposited in different rapidity region. The result supports our asymmetry argument.
\end{abstract}

Critical Point and Onset of Deconfinement - CPOD2017

7-11 August, 2017

The Wang Center, Stony Brook University, Stony Brook, NY

${ }^{*}$ Speaker. 


\section{Introduction}

In non-central heavy ion collisions, initial orbital angular momentum as large as $10^{5} \hbar$ can be generated. Part of the angular momentum is expected to be converted into the polarization of final particles through spin-orbital coupling [1,2,3]. Using $\Lambda$ hyperon's weak decay, its polarization can be measured via the angular distribution of the daughter protons [4]. Recently this topic has attracted much attention as STAR collaboration reported the observation for the global polarization of $\Lambda(\bar{\Lambda})$ hyperons in $20 \%-50 \%$ centrality Au-Au collisions in the beam energy scan (BES) program [5], see recent reviews from experimental [6] and theoretical perspective [7, 8]. The STAR's result shows that the global polarization is significantly nonzero at low collisional energies and decreases with the collisional energy in range $\sqrt{s_{N N}}=7.7 \sim 200 \mathrm{GeV}$. This energy dependence is consistent with the results from hydrodynamic and transport model's calculations $[9,10,11,12]$, but it seems contradictory to the naive expectation that the global polarization should be proportional to the total orbital angular momentum and then to the collisional energy. We have studied this problem in our previous paper [11], and attributed the polarization's energy behavior to less asymmetric participant matter in the midrapidity at higher energies. In this work, we provide further supports of this conclusion by investigating how much angular momentum is deposited in different rapidity region.

\section{Model description}

In our study, the string-melting version of A Multi-Phase Transport (AMPT) model [13] is employed to simulate the evolution of the collisional system. In the model, all participants in collisions are converted into partons. By coarse-graining the collective motion of the partons and adopting the equation of state from lattice result $[14,15]$, we obtain hydrodynamic quantities such as the fluid velocity $u^{\mu}$, the temperature $T$ and the thermal vorticity $\varpi_{\mu v}$. The thermal vorticity is defined by

$$
\varpi_{\mu v}=\frac{1}{2}\left[\partial_{v}\left(\frac{u_{\mu}}{T}\right)-\partial_{\mu}\left(\frac{u_{v}}{T}\right)\right] .
$$

In local equilibrium, $\Lambda$ 's spin four-vector is related to $\varpi_{\mu \nu}$ by $[16,17,18]$

$$
S^{\mu}=-\frac{1}{8 m}\left(1-n_{F}\right) \varepsilon^{\mu v \rho \sigma} p_{v} \varpi_{\rho \sigma}
$$

where $p^{\mu}$ and $m$ are the four-momentum and mass of the $\Lambda$ hyperon respectively, and $n_{F}=1 /[1+$ $\exp (\beta \cdot p \mp \mu / T)]$ is the Fermi-Dirac distribution function for particle (upper sign) and anti-particle (lower sign). At the end of the partonic stage in the AMPT model, all partons are converted into hadrons. Then we collect the $\Lambda(\bar{\Lambda})$ hyperons in the midrapidity region $|\eta|<1$ and calculate their spin vectors by Eq. (2.2). In the center of mass frame (c.m.f.) of two nuclei, the spin four-vector $S^{\mu}=\left(S^{0}, \mathbf{S}\right)$ has a non-zero time component $S^{0}$. To obtain the spin three-vector, we should boost $S^{\mu}$ into the $\Lambda$ 's rest frame, where the spin four-vector $S^{\mu *}$ does not have the time component, i.e. $S^{\mu *}=\left(0, \mathbf{S}^{*}\right)$ with $\mathbf{S}^{*}$ being the spin three-vector and related to $\mathbf{S}$ by

$$
\mathbf{S}^{*}=\mathbf{S}-\frac{\mathbf{p} \cdot \mathbf{S}}{E_{p}\left(m+E_{p}\right)} \mathbf{p},
$$


where $E_{p}$ and $\mathbf{p}$ are the $\Lambda$ 's energy and momentum in the c.m.f. of two nuclei. The global $\Lambda$ polarization is obtained by $P=2\left\langle\mathbf{S}^{*}\right\rangle \cdot \hat{\mathbf{J}}$, where $\hat{\mathbf{J}}$ is the unit vector along the direction of the collisional system's orbital angular momentum (perpendicular to the reaction plane), the average is taken over all $\Lambda$ hyperons, and we have introduced a normalization factor 2 (since the $\Lambda$ 's spin is $1 / 2$ ). In the AMPT's coordinate system, the $x$-axis is along the direction of impact parameter $\mathbf{b}$ and $z$ is along the beam direction. Two nuclei centered at $(x= \pm b / 2, y=0)$ move along the $\pm z$ direction, respectively. Therefore $\hat{\mathbf{J}}$ is along $-y$ direction and the global polarization is simply $P=-2\left\langle S_{y}^{*}\right\rangle$.

\section{Numerical results from AMPT}

\subsection{The global $\Lambda$ polarization}

We run the simulations at collisional energies $\sqrt{s_{N N}}=7.7,11.5,14.5,19.6,27,39,62.4$ and $200 \mathrm{GeV}$. For each energy, we chose two specific impact parameters $b=7$ and $9 \mathrm{fm}$ corresponding to $20 \%-50 \%$ centrality class in the STAR experiment. The final results are obtained by taking average over these two impact parameters.

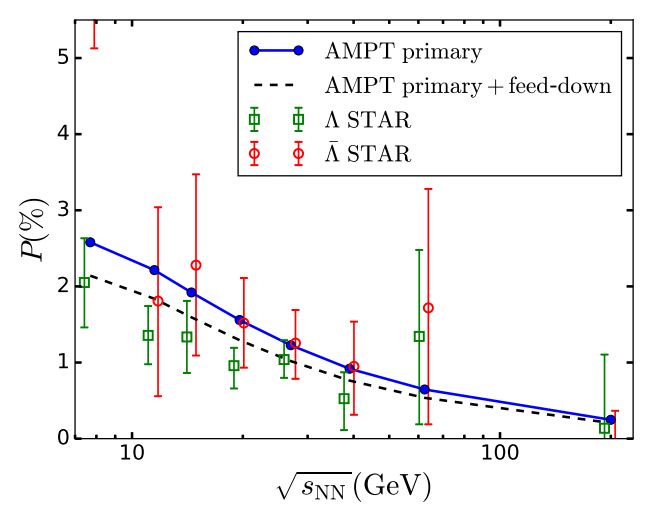

Figure 1: The energy dependence of the global $\Lambda$ polarization in the midrapidity region $|\eta|<1$ from 7.7 to $200 \mathrm{GeV}$. The curves represent the global polarization of primary $\Lambda \mathrm{s}$ (blue solid) and primary plus feeddown $\Lambda$ s (black dashed). The unfilled squares and circles represent the STAR data for the global $\Lambda$ and $\bar{\Lambda}$ polarization [5].

Fig. 1 shows the energy dependence of the global $\Lambda$ polarization in the midrapidity region $|\eta|<1$. The blue solid line counts only the $\Lambda$ hyperons produced directly from quark coalescence (known as primary $\Lambda$ ). In reality, there are also secondary $\Lambda$ s produced from the resonance decay (feed-down). These feed-down $\Lambda$ s are estimated to suppress the global polarization by about $17 \%$ from the primary-only result $[19,9,11]$. The global polarization of primary plus feed-down $\Lambda \mathrm{s}$ (black dashed line) is in agreement with the STAR's result.

We see that the global $\Lambda$ polarization decreases with the collisional energy: from about $2.1 \%$ at $7.7 \mathrm{GeV}$ to $0.2 \%$ at $200 \mathrm{GeV}$. By investigating the spatial distributions of the thermal vorticity and the $\Lambda$ hyperon's position, we give the explanation on this energy behavior in our previous paper [11]. In non-central collisions, the fireball is tilted on the reaction plane ( $x$ - $z$ plane) after 
collisions: there is more matter in $x z>0$ region than in $x z<0$ region. Such an asymmetry plays a crucial role in generating a net vorticity and the global polarization along the direction of the collisional system's angular momentum ( $-y$ direction), see Ref. [11] for details. However at high energy such as $200 \mathrm{GeV}$, the fireball extends over a wider rapidity range due to its fast longitudinal expansion. As a consequence, the tilted shape of the fireball is only visible at large rapidity, while at midrapidity the matter is almost symmetrically distributed and thus the global polarization nearly vanishes.

\subsection{Initial parton distribution and angular momentum}

We now give an illustration of the fireball's tilted shape. For simplicity, we consider the parton distribution at the initial time of the partonic phase in the AMPT model. At this time, all partons are located at $z=0$ and are distributed with their transverse positions $(x, y)$ and momenta $\left(p_{x}, p_{y}, p_{z}\right)$. Fig. 2 shows the initial parton distribution as functions of the transverse coordinate $x$ and longitudinal rapidity $Y$, where $Y$ is related to $p_{z}$ by $Y=\operatorname{arctanh}\left(p_{z} / E\right)$. In the figure we take $\sqrt{s_{N N}}=7.7,39,200 \mathrm{GeV}$ and $b=7 \mathrm{fm}$ for illustration. One can see that the parton distributions at all three energies are tilted on the reaction plane: more partons are in the region $x, Y>0$ or $x, Y<0$ in non-central collisions. Such an asymmetric parton distribution is the origin of the orbital angular momentum in the system, which can be calculated by

$$
J_{y}=-\sum_{i} x_{i} p_{z}^{i}
$$

where the sum is over all partons. One can see from Eq. (3.1) that more partons distributed in the $x p_{z}>0$ region can lead to a negative net angular momentum after taking the sum.
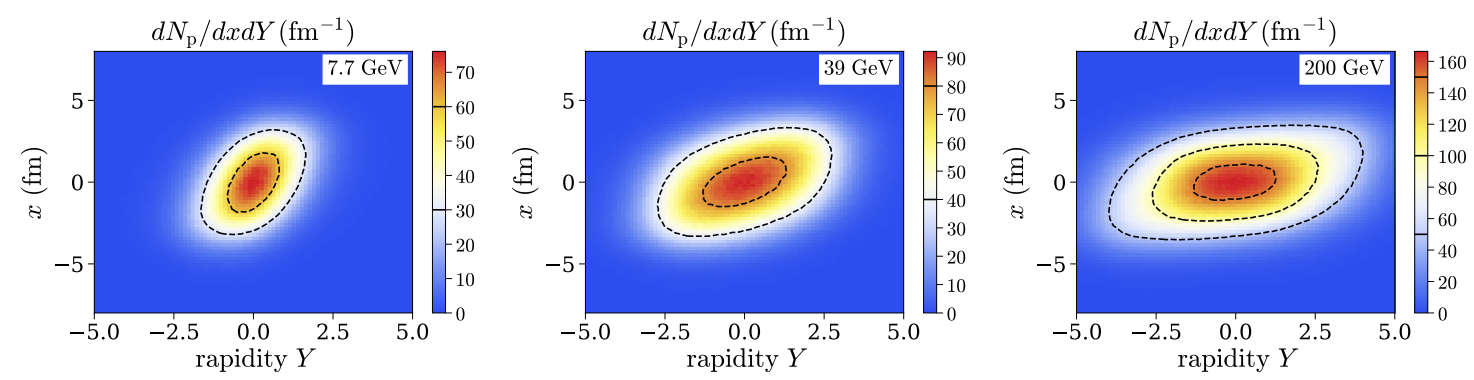

Figure 2: The initial parton distribution as functions of the transverse coordinate $x$ and longitudinal rapidity $Y$ at collisional energies $\sqrt{s_{N N}}=7.7 \mathrm{GeV}$ (left), $39 \mathrm{GeV}$ (middle) and $200 \mathrm{GeV}$ (right) and the impact parameter $b=7 \mathrm{fm}$. For better illustration, we draw equal density contours (black dashed lines) in each panel.

We also see from Fig. 2 that the parton distribution has a collisional energy and rapidity dependence. At low energies such as $7.7 \mathrm{GeV}$, the partons are distributed in smaller rapidity range with an obvious tilted shape. However at high energies like $200 \mathrm{GeV}$, the fireball shows an almost symmetric distribution at midrapidity, while the tilted shape is only visible at large rapidity due to the wider extension of the fireball. The collisional energy and rapidity dependence of parton distributions can lead to the same dependence of the orbital angular momentum.

Fig. 3 shows how the angular momentum is deposited in different rapidity bins at three collisional energies, where the width of the rapidity bin is set to be one unit. For each collisional energy, 

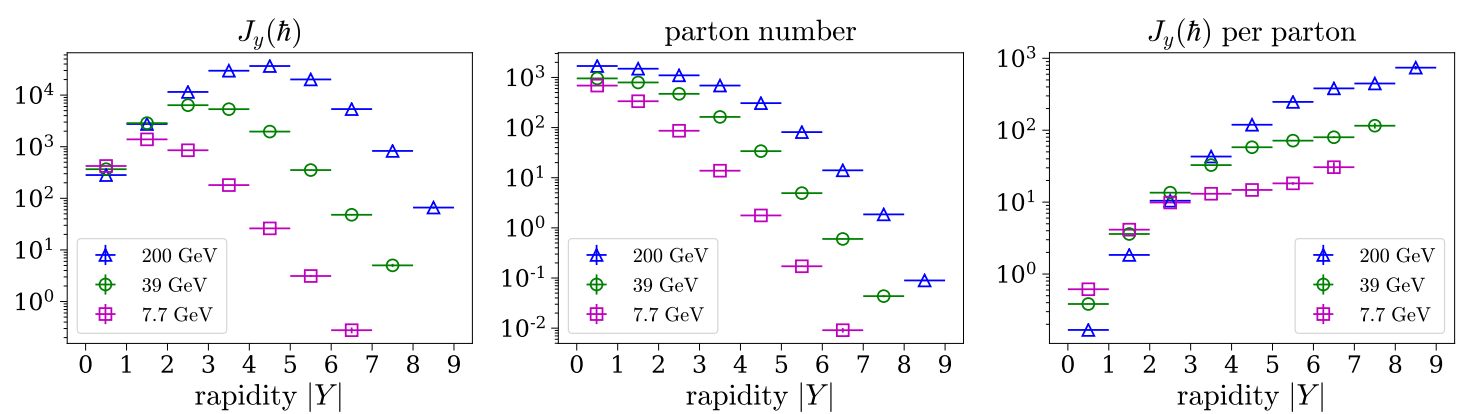

Figure 3: The orbital angular momentum $J_{y}$ carried by partons (left), the parton number (middle), and the orbital angular momentum $J_{y}$ per parton (right) in different rapidity bins at collisional energies $\sqrt{s_{N N}}=7.7$ $\mathrm{GeV}$ (magenta square), $39 \mathrm{GeV}$ (green circle) and $200 \mathrm{GeV}$ (blue triangle) with the impact parameter $b=7$ $\mathrm{fm}$. Note that in our coordinate system $J_{y}$ is always negative, but values in the figures are its magnitudes without the sign.

$10^{4}$ events are generated for statistics. In the left panel, the shapes of the angular momentum are similar for each energy. $J_{y}$ first increases with rapidity due to the increase of both $\left|p_{z}\right|$ and the asymmetry, and then decreases at larger rapidity. The decrease behavior is because of less partons at larger rapidity as shown in the middle panel. To remove the effect of decreasing parton number with rapidity, we divide $J_{y}$ by the parton number in each bin. The result shown in the right panel turns out to increase with rapidity monotonically.

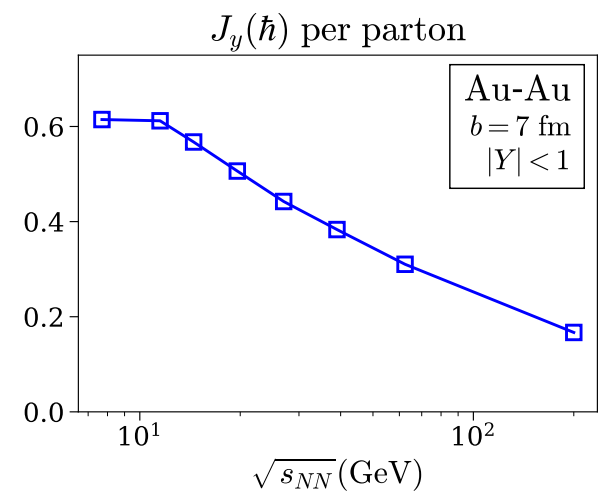

Figure 4: The orbital angular momentum $J_{y}$ per parton in the rapidity bin $|Y|<1$ as a function of the collisional energy from 7.7 to $200 \mathrm{GeV}$.

Since more partons with larger $\left|p_{z}\right|$ are generated in higher energy collisions, the total angular momentum in the system should increase with the collisional energy, see Refs. [20, 9] for example. However, such an energy behavior is not true for the angular momentum deposited in the midrapidity region. Actually, as shown in Fig. 3, most of the total angular momentum is carried away by partons in larger rapidity. In the middle rapidity bin $|Y|<1$, Fig. 4 shows the angular momentum per parton increases with decreasing collisional energy. This energy behavior is the same with the energy dependence of the global polarization [5] and the directed flow slope $d v_{1} / d \eta$ [6]. It indicates that the hot and dense matter created in non-central collisions is more tilted at lower energies in the midrapidity region. 
We note that the curve in Fig. 4 is almost flat between 7.7 and $11.5 \mathrm{GeV}$, although more tilted parton distribution on the reaction plane is preferred at $7.7 \mathrm{GeV}$. This can be understood by that the parton density $d N_{p} / d Y$ sharply decreases with rapidity at $7.7 \mathrm{GeV}$, making the mean value of $\left|p_{z}\right|$ smaller than that at higher energies.

\section{Summary}

We have studied the vorticity-induced polarization of the $\Lambda$ hyperons in the midrapidity region for Au-Au collisions at $\sqrt{s_{N N}}=7.7 \sim 200 \mathrm{GeV}$ with the AMPT model. Our result is in good agreement with the recent STAR measurement. The value of global $\Lambda$ polarization at $7.7 \mathrm{GeV}$ is about one order of magnitude larger than that of $200 \mathrm{GeV}$. We also investigate how the angular momentum is distributed in the system. The result shows that although the angular momentum in the whole system increases with collisional energy, the one deposited in the midrapidity per parton decreases on the contrary. The latter energy behavior is the same with the global polarization, and indicates the participant matter in non-central collisions is more tilted at lower energies in the midrapidity.

\section{Acknowledgements}

The authors are supported in part by the Major State Basic Research Development Program (973 Program) in China under the Grant No. 2015CB856902 and 2014CB845402 and by the National Natural Science Foundation of China (NSFC) under the Grant No. 11535012.

\section{References}

[1] Z.-T. Liang and X.-N. Wang, Globally polarized quark-gluon plasma in non-central A+A collisions, Phys. Rev. Lett. 94 (2005) 102301, [nucl-th/ 0410079 ].

[2] S. A. Voloshin, Polarized secondary particles in unpolarized high energy hadron-hadron collisions?, nucl-th/0410089.

[3] B. Betz, M. Gyulassy and G. Torrieri, Polarization probes of vorticity in heavy ion collisions, Phys. Rev. C76 (2007) 044901, [0 708 . 0 035].

[4] STAR collaboration, B. I. Abelev et al., Global polarization measurement in Au+Au collisions, Phys. Rev. C76 (2007) 024915, [0 705.1691$]$.

[5] STAR collaboration, L. Adamczyk et al., Global $\Lambda$ hyperon polarization in nuclear collisions: evidence for the most vortical fluid, Nature 548 (2017) 62-65, [1701.06657].

[6] S. A. Voloshin, Vorticity and particle polarization in heavy ion collisions (experimental perspective), in 17th International Conference on Strangeness in Quark Matter (SQM 2017) Utrecht, the Netherlands, July 10-15, 2017, 2017, 1710.08934 , https://inspirehep.net/record/1632493/files/arXiv:1710.08934.pdf.

[7] F. Becattini, Polarization in relativistic heavy ion collisions: a theoretical perspective, in 17th International Conference on Strangeness in Quark Matter (SQM 2017) Utrecht, the Netherlands, July 10-15, 2017, 2017, 1711.08780, https://inspirehep.net/record/1638420/files/arXiv:1711.08780.pdf. 
[8] Q. Wang, Global and local spin polarization in heavy ion collisions: a brief overview, Nucl. Phys. A967 (2017) 225-232, [1704.04022].

[9] I. Karpenko and F. Becattini, Study of $\Lambda$ polarization in relativistic nuclear collisions at $\sqrt{s_{\mathrm{NN}}}=7.7$ -200 GeV, Eur. Phys. J. C77 (2017) 213, [1610 . 04717].

[10] Y. Xie, D. Wang and L. P. Csernai, Global $\Lambda$ polarization in high energy collisions, Phys. Rev. $\mathbf{C 9 5}$ (2017) 031901, [1703.03770].

[11] H. Li, L.-G. Pang, Q. Wang and X.-L. Xia, Global $\Lambda$ polarization in heavy-ion collisions from a transport model, Phys. Rev. C96 (2017) 054908, [1704.01507].

[12] Y. Sun and C. M. Ko, $\Lambda$ hyperon polarization in relativistic heavy ion collisions from a chiral kinetic approach, Phys. Rev. C96 (2017) 024906, [1706.09467].

[13] Z.-W. Lin, C. M. Ko, B.-A. Li, B. Zhang and S. Pal, A Multi-phase transport model for relativistic heavy ion collisions, Phys. Rev. C72 (2005) 064901, [nucl-th/ 0411110 ].

[14] S. Borsanyi, G. Endrodi, Z. Fodor, S. D. Katz, S. Krieg, C. Ratti et al., QCD equation of state at nonzero chemical potential: continuum results with physical quark masses at order $\mathrm{mu}^{2}, J H E P(08$ (2012) 053, [1204.6710].

[15] A. Bazavov et al., The QCD Equation of State to $\mathscr{O}\left(\mu_{B}^{6}\right)$ from Lattice QCD, Phys. Rev. D95 (2017) 054504, [1701.04325].

[16] F. Becattini and F. Piccinini, The Ideal relativistic spinning gas: Polarization and spectra, Annals Phys. 323 (2008) 2452-2473, [0710.5694].

[17] F. Becattini, V. Chandra, L. Del Zanna and E. Grossi, Relativistic distribution function for particles with spin at local thermodynamical equilibrium, Annals Phys. 338 (2013) 32-49, [1303. 3431 ].

[18] R.-h. Fang, L.-g. Pang, Q. Wang and X.-n. Wang, Polarization of massive fermions in a vortical fluid, Phys. Rev. C94 (2016) 024904, [1604.04036].

[19] F. Becattini, I. Karpenko, M. Lisa, I. Upsal and S. Voloshin, Global hyperon polarization at local thermodynamic equilibrium with vorticity, magnetic field and feed-down, Phys. Rev. C95 (2017) 054902, [1610.02506].

[20] Y. Jiang, Z.-W. Lin and J. Liao, Rotating quark-gluon plasma in relativistic heavy ion collisions, Phys. Rev. C94 (2016) 044910, [1602.06580]. 